\title{
Prevalência e fatores associados à depressão entre idosos institucionalizados: subsídio ao cuidado de enfermagem*
}

\author{
PREVALENCE AND FACTORS ASSOCIATED WITH DEPRESSION AMONG \\ INSTITUTIONALIZED ELDERLY INDIVIDUALS: NURSING CARE SUPPORT
}

\author{
PREVALENCIA Y FACTORES ASOCIADOS A LA DEPRESIÓN EN ANCIANOS \\ INSTITUCIONALIZADOS: SOPORTE A LAATENCIÓN DE ENFERMERÍA
}

\section{Elisa Roesler e Silva ${ }^{1}$, Allana Resende Pimentel Sousa ${ }^{2}$, Luzitano Brandão Ferreira ${ }^{3}$, Henry Maia Peixoto ${ }^{4}$}

\section{RESUMO}

Este estudo teve como objetivo a verificação de depressão entre idosos institucionalizados. Empregou-se o método transversal utilizando-se a Escala de Depressão Geriátrica de Yesavage em cinco instituições de longa permanência do Distrito Federal para verificar sintomas de depressão. Foram estudados ao todo 299 indivíduos. Destes, 181 não atenderam os critérios de inclusão e 16 se recusaram a participar do estudo. Dos 102 idosos com condições de participar do estudo, 49,0\% apresentavam depressão. Destes, $36,3 \%$ com depressão leve a moderada e $12,7 \%$ com depressão severa. Verificou-se associações entre sintomas de depressão e aumento da idade, sexo feminino, limitação/dependência e insatisfação com a instituição. Houve ainda associação significativa entre depressão e insônia, taquicardia, parestesia, tontura e suor excessivo. A depressão é altamente prevalente entre idosos institucionalizados, é mais comum entre as mulheres, e relaciona-se a uma série de sinais e sintomas que podem auxiliar em um diagnóstico precoce, subsidiando uma assistência de enfermagem mais efetiva.

\section{DESCRITORES}

Depressão

Idoso

Instituição de longa permanência para idosos Saúde do idoso institucionalizado

\begin{abstract}
The objective of this study was to verify depression among institutionalized elderly individuals. The cross-section method and Yesavage's Geriatric Depression Scale were utilized in five geriatric long-term care facilities located in the Federal District to verify symptoms of depression. A total of 299 individuals were studied, 181 of whom did not meet the inclusion criteria. Sixteen individuals declined to participate. Considering the 102 individuals found suitable to participate in the study, $49.0 \%$ presented symptoms of depression; $36.3 \%$ suffered from mild to moderate depression and $12.7 \%$ showed symptoms of severe depression. Associations were found between depression symptoms and increased age, female gender, physical limitations/dependence and dissatisfaction with the institution. A significant association was also found between depression and insomnia, tachycardia, paresthesias, dizziness and excessive sweating. Depression is highly prevalent among institutionalized elderly individuals, more common among women, and correlated with a series of signs and symptoms that may help in making an early diagnosis, thus offering support to providing more effective nursing care.
\end{abstract}

DESCRIPTORS
Depression
Aged
Homes for the Aged
Health of institutionalized elderly

\section{RESUMEN}

Se objetivó verificar la depresión en ancianos institucionalizados. Se empleó el método transversal, utilizando la Escala de Depresión Geriátrica de Yesavage en cinco hogares geriátricos del Distrito Federal, para verificar sintomatología depresiva. Fueron estudiados en total 299 individuos, 181 no atendieron los criterios de inclusión y 16 recusaron su participación. De los 102 ancianos participantes del estudio, 49,0\% presentaba depresión: $36,3 \%$ de leve a moderada, $12,7 \%$ depresión severa. Se verificaron asociaciones entre síntomas depresivos y aumento etario, sexo femenino, limitación/dependencia e insatisfacción con la institución. Existió inclusive asociación significativa entre depresión e insomnio, taquicardia, parestesia, mareos y sudoración excesiva. La depresión es altamente prevalente entre ancianos institucionalizados, es más común entre las mujeres, y se relaciona a una serie de signos y síntomas que pueden cooperar a un diagnóstico precoz, facilitándose una atención de enfermería más efectiva.

\author{
DESCRIPTORES \\ Depresión \\ Anciano \\ Hogares para ancianos \\ Salud del anciano institucionalizado
}

*Extraído da monografia de conclusão de curso "Depressão entre idosos residentes em instituições de longa permanência do Distrito Federal”, Graduação em Enfermagem, Faculdade de Ciências da Saúde, Centro Universitário de Brasília, 2011. "Enfermeira pela Faculdade de Ciências da Saúde do Centro Universitário de Brasília. Brasília, DF, Brasil. elisaroesler@yahoo.com.br ${ }^{2}$ Enfermeira pela Faculdade de Ciências da Saúde do Centro Universitário de Brasília. Brasília, DF, Brasil. allana2rps@yhaao.com.br ${ }^{3}$ Médico. Doutor pela Faculdade de Ciências da Saúde do Centro Universitário de Brasília. Brasília, DF, Brasil. luzitano.ferreira@uniceub.br ${ }^{4}$ Enfermeiro. Mestrando do Programa de Pós-Graduação em Enfermagem da Universidade de Brasília. Brasília, DF, Brasil. henry.peixoto@uniceub.br 


\section{INTRODUÇÃO}

O envelhecimento humano pode ser definido como as alterações morfofuncionais que leva o indivíduo a um processo contínuo e irreversível de desestruturação orgânica. Abrange fatores hereditários, ação do meio ambiente, a própria idade, dieta, tipo de ocupação, estilo de vida, dentre outros, todos condicionados pelo contexto social ao qual o indivíduo pertence ${ }^{(1)}$.

Nos últimos anos, com o envelhecimento populacional a temática do idoso tem ganhado relevância, especialmente pelas doenças apresentadas por esta faixa etária da população, incluindo um elevado número de doenças psiquiátricas, especialmente a depressão(2).

A Organização Mundial de Saúde considera a depressão um grave problema de saúde pública e estima que 154 milhões de pessoas sejam afetadas em todo mundo ${ }^{(3)}$. A prevalência de depressão entre os idosos pode variar muito, dependendo do instrumento utilizado e da gravidade estudada ${ }^{(4)}$. Estima-se que $15 \%$ dos idosos apresentam algum sintoma depressivo, e que a depressão seja frequente em idosos hospitalizados (5 a 13\%) e institucionalizados $(12 \text { a } 16 \%)^{(5)}$.

$O$ idoso com sintomas de depressão frequentemente é negligenciado quanto ao diagnóstico e ao tratamento da depressão, o que altera sua qualidade de vida, além de levar ao aumento da carga econômica aos serviços de saúde, por seus custos diretos e indiretos ${ }^{(6)}$. Apesar de sua relevância clíni$\mathrm{ca}$, a sintomatologia depressiva em idosos é pouco verificada e valorizada por parte dos profissionais de saúde ${ }^{(7)}$.

berang

Apesar de sua relevância clínica, a sintomatologia depressiva em idosos é pouco verificada e valorizada por parte dos profissionais de saúde.
Nesta perspectiva, a enfermagem pode exercer um papel fundamental, oferecendo uma práxis voltada ao enveIhecimento saudável, compreendendo os fenômenos como eles se apresentam e assegurando o atendimento das necessidades do idoso, a fim de preservar a sua saúde física e mental e o aperfeiçoamento moral, intelectual, espiritual, em condições de autonomia e dignidade. Assim, a abordagem da depressão geriátrica pela enfermagem, deve ir além de uma abordagem convencional com enfoque curativo, dirigindo-se a promoção da saúde mental e a prevenção da depressão, prescindindo, portando, da identificação adequada do agravo e fatores de risco relacionados ${ }^{(8-10)}$.

Estudos sobre depressão em idosos mostram-se relevantes na prática clínica, pois possibilitam intervenções precoces e efetivas, além da prevenção de fatores de ris$\mathrm{co}^{(4,6)}$. Nesta perspectiva, a avaliação da sintomatologia depressiva por meio da aplicação de escalas reconhecidas internacionalmente pode contribuir para melhor detectar os casos de depressão nessa faixa etária ${ }^{(7)}$. O presente estudo teve como objetivo avaliar a prevalência de sintomas de depressão em idosos institucionalizados e verificar possíveis fatores associados, além de verificar possíveis fatores associados que possam subsidiar a assistência de enfermagem.

\section{MÉTODO}

O presente estudo apresentou um delineamento observacional, com metodologia seccional do tipo corte transversal, realizado em cinco Instituições de Longa Permanência para Idosos, localizadas no Distrito Federal.

A Escala de Depressão Geriátrica em versão reduzida de Yesavage (EDG-15) foi utilizada para detecção de sinais indicativos de depressão nos idosos. Foi realizado um estudo de rastreamento da presença de sinais indicativos de depressão e não de uma avaliação diagnóstica clínica. A escala de depressão apresenta 15 perguntas negativas/ afirmativas que verifica sintomas depressivos, no qual uma pontuação entre 0 e 5 se considera normal, 6 a 10 indica depressão leve a moderada e 11 a 15 depressão severa.

Os critérios de inclusão foram: idosos com 60 anos ou mais, de ambos os sexos, com capacidade cognitiva para responder o questionário, sem demência ou doenças psiquiátricas prévias (verificadas através do prontuário médico), que aceitaram participar do estudo e assinaram o Termo de Consentimento Livre e Esclarecido.

Os idosos que consentiram em participar da pesquisa responderam a um questionário dividido em três partes: na primeira parte foram verificados aspectos sócio-econômicos como: sexo, idade, estado civil, cor (auto-definida), grau de escolaridade e religião. $\mathrm{Na}$ segunda parte foram avaliados aspectos que poderiam influenciar no desencadeamento da depressão em idosos, como uso de medicamentos, grau de limitação/dependência e tempo de instituição. Na terceira parte foram avaliados sinais e sintomas pré-determinados (insônia, dor, taquicardia, tontura, suor excessivo) que poderiam associar-se à depressão.

A análise estatística dos dados foi realizada no programa Epi Info, versão 3.5.1. Foram utilizados os testes de Qui-Quadrado e Exato de Fisher para a associação entre variáveis categóricas independentes e o teste Mann-Whitney, depois de constatada a não normalidade das distribuições das frequências, para variáveis numéricas e categóricas, levando em consideração o nível de significância de $5 \%(p<0,05)$.

Todos os participantes assinaram o Termo de Consentimento Livre e Esclarecido (TCLE) e a presente pesquisa foi aprovada pelo Comitê de Ética em Pesquisa da Fundação de Ensino e Pesquisa em Ciências da Saúde (CEP-FEPECS), do Governo do Distrito Federal (protocolo n. 191/10).

A primeira instituição onde foram avaliados os idosos apresenta caráter filantrópico, atende indivíduos de am- 
bos os sexos, sem condições de auto-sustento em regime de longa permanência. A estrutura física é dividida em ala feminina e ala masculina constituídos por quartos triplos. A equipe de saúde é constituída por profissionais contratados pela instituição (cuidadores e terapeuta ocupacional) e por profissionais voluntários (médico, nutricionista, e psicólogo).

A segunda instituição, também filantrópica, atende idosos de baixa renda, dependentes ou independentes, em regime de longa permanência. O lar dispõe de cuidadores contratados pela instituição e profissionais voluntários (médico e nutricionista). Os quartos são divididos por no máximo dois idosos.

A terceira instituição é privada, atende idosos de ambos os sexos, dependentes e independentes, em regime de internato ou semi-internato. Dispõe de equipe multidisciplinar contratada pela instituição (médico geriatra, enfermeira, nutricionistas e técnicos de enfermagem). Possui cômodos individuais e duplos.

A quarta instituição, de caráter privado, atende idosos independentes e dependentes, em regime de internato ou semi-internato. Possui atendimento médico geriátrico e assistência de enfermagem prestada por enfermeiro e técnicos de enfermagem. A instituição conta também com nutricionistas, fisioterapeutas e uma terapeuta ocupacional. A estrutura física é constituída por cômodos individuais, duplos, triplos e quádruplos.

A quinta instituição, também privada, atende idosos, independentemente da renda, com e sem dependência. Dispõem de quartos duplos. Contam com uma equipe de cuidadores contratados e com a ajuda voluntária de médicos, enfermeiros, fisioterapeutas e nutricionistas.

\section{RESULTADOS}

Dos 299 indivíduos institucionalizados pesquisados, 181 não atenderam os critérios de inclusão. Dois tinham idade inferior a 60 anos e 179 não apresentaram condições clínicas para responder o questionário devido à demência, rebaixamento do nível de consciência ou patologias psiquiátricas prévias (verificadas através de revisão de prontuários). Após a exclusão dos idosos que não atendiam os critérios de inclusão, 118 idosos foram incluídos na pesquisa. Destes 16 se recusaram a participar do estudo, ficando a presente amostra com o número final de 102 participantes.

A Tabela 1 especifica as características sócio-demográficas dos indivíduos estudados. Houve uma predominância do sexo feminino $(60,8 \%)$, de idosos com idade acima de 80 anos $(41,2 \%)$, da cor branca $(43,1 \%)$ e de solteiros $(35,3 \%)$. A média de anos de estudo da população foi de 5,6 anos e $84,3 \%$ dos indivíduos recebem aposentadoria.

Dos indivíduos avaliados, 50 (49,0\%) apresentavam depressão. Destes, 37 (36,3\%) foram classificados como apresentando depressão leve e 13 (12,7\%) depressão severa.

As verificações de associações entre a depressão e as variáveis demográficas e socioeconômicas (Tabela 1), foram significantes para idade $(p=0,04)$ e sexo $(p=0,01)$. A proporção de indivíduos com depressão, bem como sua gravidade, teve aumento proporcional com o aumento da idade. A depressão severa, que correspondeu a zero na faixa etária entre 60-69 anos, aumentou para 14,3\% entre indivíduos com $70-80$ anos e para $19,0 \%$ entre os idosos acima de 80 anos. Houve uma maior prevalência de mulheres com depressão. Destas 19,4\% apresentaram depressão severa e $38,7 \%$ leve. Entre os homens foi observado 2,5\% de depressão severa e 32,5\% de depressão leve.

Tabela 1 - Distribuição dos idosos residentes em instituições de longa permanência do Distrito Federal, segundo diagnóstico de depressão, classificação da Escala de Depressão Geriátrica (EDG-15) e variáveis demográficas e socioeconômicas do Distrito Federal em 2010

\begin{tabular}{|c|c|c|c|c|c|c|c|c|}
\hline & Total & SD & CD & \multirow{2}{*}{$\mathbf{p}$} & DepS & DepLM & Normal & \multirow{2}{*}{$\mathbf{P}$} \\
\hline & f $\%$ & $\%$ & $\%$ & & f $\%$ & $\%$ & $\%$ & \\
\hline \multicolumn{9}{|l|}{ Idade } \\
\hline $60-70$ & $25(24,5)$ & $17(68,0)$ & $8(32,0)$ & $0,025^{*}$ & $0(0,0)$ & $8(32,0)$ & $17(68,0)$ & $0,044^{\mathrm{a}}$ \\
\hline $71-80$ & $35(34,3)$ & $20(57,1)$ & $15(42,9)$ & & $5(14,3)$ & $10(28,6)$ & $20(57,1)$ & \\
\hline$>80$ & $42(41,2)$ & $15(35,7)$ & $27(64,3)$ & & $8(19,0)$ & $19(45,2)$ & $15(35,7)$ & \\
\hline \multicolumn{9}{|l|}{ Sexo } \\
\hline Feminino & $60(60,8)$ & $26(41,9)$ & $36(58,1)$ & $0,019^{* *}$ & $12(19,4)$ & $24(38,7)$ & $26(41,9)$ & $0,016^{\mathrm{a}}$ \\
\hline Masculino & $40(39,2)$ & $26(65,0)$ & $14(35,0)$ & & $1(2,5)$ & $13(32,5)$ & $26(65,0)$ & \\
\hline \multicolumn{9}{|l|}{ Estado civil } \\
\hline Casado & $13(12,7)$ & $8(61,1)$ & $5(38,5)$ & $0,134^{*}$ & $1(7,7)$ & $4(30,8)$ & $8(61,5)$ & $0,277^{\mathrm{a}}$ \\
\hline Divorciado & $17(16,7)$ & $11(64,7)$ & $6(35,3)$ & & $2(11,8)$ & $4(23,5)$ & $11(64,7)$ & \\
\hline Namorando & $1(1,0)$ & $1(100)$ & $0(0,0)$ & & $0(0,0)$ & $0(0,0)$ & $1(100)$ & \\
\hline Solteiro & $36(35,3)$ & $20(55,6)$ & $16(44,4)$ & & $2(5,6)$ & $14(38,9)$ & $20(56,6)$ & \\
\hline Viúvo & $35(34,3)$ & $12(34,3)$ & $23(65,7)$ & & $8(22,9)$ & $15(42,9)$ & $12(34,3)$ & \\
\hline \multicolumn{9}{|l|}{ Aposentado } \\
\hline Não & $16(15,7)$ & $7(43,8)$ & $9(56,3)$ & $0,360^{* *}$ & $5(31,3)$ & $4(25,0)$ & $7(43,8)$ & $0,051^{\mathrm{a}}$ \\
\hline Sim & $86(84,3)$ & $45(52,3)$ & $41(47,7)$ & & $8(9,3)$ & $33(38,4)$ & $45(52,3)$ & \\
\hline
\end{tabular}

ateste qui-quadrado. Nota: sd: sem depressão; cd: com depressão; deplm: depressão leve/moderada; deps: depressão severa. 
Com relação a possíveis fatores interferentes na depressão de idosos institucionalizados (Tabela 2), a análise dos resultados demonstrou associação significativa com a existência de algum tipo de limitação/dependência $(p=0,01)$ e insatisfação do idoso com a instituição na que reside $(p=0,002)$. Entre os idosos com depressão, $60,4 \%$ possuem algum tipo de limitação/dependência física e $80,8 \%$ se dizem insatisfeitos com a instituição que residem.

Tabela 2 - Principais fatores relacionados com a depressão de idosos residentes em instituições de longa permanência do Distrito Federal em 2010

\begin{tabular}{|c|c|c|c|c|c|c|c|}
\hline \multirow{2}{*}{ Variáveis } & \multicolumn{2}{|c|}{ Total } & \multicolumn{2}{|c|}{ Sem Depressão } & \multicolumn{2}{|c|}{ Com Depressão } & \multirow{2}{*}{$\mathbf{P}$} \\
\hline & $\mathrm{f}$ & $\%$ & $\mathrm{f}$ & $\%$ & $\mathrm{f}$ & $\%$ & \\
\hline Religião & & & & & & & $0,6201^{\mathrm{b}}$ \\
\hline Não & 8 & $(7,8)$ & 4 & $(50,0)$ & 4 & $(50,0)$ & \\
\hline Sim & 94 & $(92,2)$ & 48 & $(51,1)$ & 46 & $(48,9)$ & \\
\hline Categoria da Religião & & & & & & & $0.8348^{\mathrm{a}}$ \\
\hline Católica & 68 & $(66,7)$ & 35 & $(51,5)$ & 33 & $(48,5)$ & \\
\hline Espírita & 8 & $(7,8)$ & 5 & $(62,5)$ & 3 & $(37,5)$ & \\
\hline Evangélica & 15 & $(14,7)$ & 6 & $(40,0)$ & 9 & $(60,0)$ & \\
\hline Outra & 3 & $(2,9)$ & 2 & $(66,7)$ & 1 & $(33,3)$ & \\
\hline Recebe visita & & & & & & & $0,4605^{\mathrm{b}}$ \\
\hline Não & 28 & $(27,5)$ & 15 & $(53,6)$ & 13 & $(46,4)$ & \\
\hline Sim & 74 & $(72,5)$ & 37 & $(50,0)$ & 37 & $(50,0)$ & \\
\hline Limitação/dependência & & & & & & & $0,0141^{\mathrm{b}}$ \\
\hline Não & 49 & $(48,0)$ & 31 & $(63,3)$ & 18 & $(36,7)$ & \\
\hline Sim & 53 & $(52,0)$ & 21 & $(39,6)$ & 32 & $(60,4)$ & \\
\hline Atividade física & & & & & & & $0,1627^{\mathrm{b}}$ \\
\hline Não & 49 & $(48,0)$ & 22 & $(44,9)$ & 27 & $(55,1)$ & \\
\hline Sim & 53 & $(52,0)$ & 30 & $(56,6)$ & 23 & $(43,4)$ & \\
\hline Doença & & & & & & & $0,3646^{\mathrm{b}}$ \\
\hline Não & 25 & $(24,5)$ & 14 & $(56,0)$ & 11 & $(44,0)$ & \\
\hline Sim & 77 & $(75,5)$ & 38 & $(49,4)$ & 39 & $(50,6)$ & \\
\hline Faz uso de medicação & & & & & & & $0,0539^{\mathrm{b}}$ \\
\hline Não & 15 & $(14,7)$ & 11 & $(73,3)$ & 4 & $(26,7)$ & \\
\hline Sim & 87 & $(85,3)$ & 41 & $(47,1)$ & 46 & $(52,9)$ & \\
\hline Sai da instituição & & & & & & & $0,3123^{\mathrm{b}}$ \\
\hline Não & 68 & $(66,7)$ & 33 & $(48,5)$ & 35 & $(51,5)$ & \\
\hline Sim & 34 & $(33,3)$ & 19 & $(55,9$ & 15 & $(44,1)$ & \\
\hline Anos de instituição & & & & & & & $0.6417^{\mathrm{a}}$ \\
\hline Até 5 anos & 63 & $(61,8)$ & 34 & $(54,0)$ & 29 & $(46,0)$ & \\
\hline 5 a 10 anos & 22 & $(21,6)$ & 11 & $(50,0)$ & 11 & $(50,0)$ & \\
\hline Mais de 10 anos & 17 & $(16,7)$ & 7 & $(41,2)$ & 10 & $(58,8)$ & \\
\hline Satisfeito com instituição & & & & & & & $0,0002^{\mathrm{b}}$ \\
\hline Não & 26 & $(25,5)$ & 5 & $(19,2)$ & 21 & $(80,8)$ & \\
\hline Sim & 76 & $(74,5)$ & 47 & $(61,8)$ & 29 & $(38,2)$ & \\
\hline Participa das atividades & & & & & & & $0,2787^{\mathrm{b}}$ \\
\hline Não & 49 & $(48,0)$ & 23 & $(46,9)$ & 26 & $(53,1)$ & \\
\hline Sim & 53 & $(52,0)$ & 29 & $(54,7)$ & 24 & $(45,3)$ & \\
\hline Gostaria de estar com a família & & & & & & & $0,1538^{\mathrm{b}}$ \\
\hline Não & 26 & $(25,5)$ & 16 & $(61,5)$ & 10 & $(38,5)$ & \\
\hline Sim & 76 & $(74,5)$ & 36 & $(47,4)$ & 40 & $(52,6)$ & \\
\hline Anos sem trabalhar & & & & & & & $0.781^{\mathrm{a}}$ \\
\hline Até 5 anos & 6 & $(5,9)$ & 3 & $(50,0)$ & 3 & $(50,0)$ & \\
\hline 5 a 10 anos & 11 & $(10,8)$ & 7 & $(63,6)$ & 4 & $(36,4)$ & \\
\hline Mais de 10 anos & 43 & $(42,2)$ & 20 & $(46,5)$ & 23 & $(53,5)$ & \\
\hline Não lembra & 42 & $(41,2)$ & 22 & $(52,4)$ & 20 & $(47,6)$ & \\
\hline
\end{tabular}

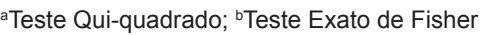

A Tabela 3 apresenta a distribuição da depressão de acordo com as cinco instituições pesquisadas. Não houve associação estatisticamente significante entre residir nas instituições e sintomas de depressão. 
Tabela 3 - Presença de depressão entre idosos residentes em diferentes instituições de longa permanência do Distrito Federal em 2010

\begin{tabular}{lcccccc}
\hline \multicolumn{1}{c}{ Variável } & $\begin{array}{c}\text { Inst 1 } \\
\text { f (\%) }\end{array}$ & $\begin{array}{c}\text { Inst 2 } \\
\text { f (\%) }\end{array}$ & $\begin{array}{c}\text { Inst 3 } \\
\mathbf{f ( \% )}\end{array}$ & $\begin{array}{c}\text { Inst 4 } \\
\mathbf{f}(\mathbf{\%})\end{array}$ & $\begin{array}{c}\text { Inst 5 } \\
\mathbf{f}(\mathbf{\%})\end{array}$ & P \\
\hline Sem depressão & $13(52)$ & $17(48,6)$ & $2(50)$ & $8(66,6)$ & $12(46,1)$ \\
Depressão Leve & $12(48)$ & $11(31,4)$ & $2(50)$ & $2(16,7)$ & $10(38,5)$ \\
Depressão Severa & $0(0)$ & $7(20)$ & $0(0)$ & $2(16,7)$ & $4(15,4)$ & \\
Total & $25(100)$ & $35(100)$ & $4(100)$ & $12(100)$ & $26(100)$ & $0,354^{\mathrm{a}}$ \\
\hline
\end{tabular}

${ }^{\text {aTTe }}$ Qui-quadrado. Nota: Inst: Instituição.

A Tabela 4 mostra uma associação significativa entre insônia $(p<0,01)$, taquicardia $(p=0,01)$, parestesia $(p<0,01)$, tontura $(p<0,01)$, suor excessivo $(p<0,01)$ e depressão. Entre os idosos deprimidos, a prevalência dos sintomas variou de $65 \%$ (dor) a $88,2 \%$ (suor excessivo) enquanto entre os não deprimidos, variou entre $11,8 \%$ (suor excessivo) e $35 \%$ (dor).

Tabela 4 - Distribuição das queixas clínicas dos idosos residentes em instituições de longa permanência do Distrito Federal em 2010

\begin{tabular}{|c|c|c|c|c|c|c|c|}
\hline \multirow{2}{*}{ Variáveis } & \multicolumn{2}{|c|}{ Total } & \multicolumn{2}{|c|}{ Sem Depressão } & \multicolumn{2}{|c|}{ Com Depressão } & \multirow{2}{*}{$\mathbf{p}$} \\
\hline & $\mathrm{F}$ & $\%$ & $\mathrm{~F}$ & $\%$ & $\mathrm{f}$ & $\%$ & \\
\hline Insônia & & & & & & & $0,0001^{\mathrm{b}}$ \\
\hline Não & 62 & $(60,8)$ & 41 & $(66,1)$ & 21 & $(33,9)$ & \\
\hline Sim & 40 & $(39,2)$ & 11 & $(27,5)$ & 29 & $(72,5)$ & \\
\hline Taquicardia & & & & & & & $0,0125^{\mathrm{b}}$ \\
\hline Não & 85 & $(83,3)$ & 48 & $(56,5)$ & 37 & $(43,5)$ & \\
\hline Sim & 17 & $(16,7)$ & 4 & $(23,5)$ & 13 & $(76,5)$ & \\
\hline Dor & & & & & & & $0,0001^{\mathrm{b}}$ \\
\hline Não & 42 & $(41,2)$ & 31 & $(73,8)$ & 11 & $(26,2)$ & \\
\hline Sim & 60 & $(58,8)$ & 21 & $(35,0)$ & 39 & $(65,0)$ & \\
\hline Parestesia & & & & & & & $0,0069^{b}$ \\
\hline Não & 84 & $(82,4)$ & 48 & $(57,1)$ & 36 & $(42,9)$ & \\
\hline Sim & 18 & $(17,6)$ & 4 & $(22,2)$ & 14 & $(77,8)$ & \\
\hline Tontura & & & & & & & $0,0009^{b}$ \\
\hline Não & 69 & $(67,6)$ & 43 & $(62,3)$ & 26 & $(37,7)$ & \\
\hline Sim & 33 & $(32,4)$ & 9 & $(27,3)$ & 24 & $(72,7)$ & \\
\hline Suor excessivo & & & & & & & $0,0003^{b}$ \\
\hline Não & 85 & $(83,3)$ & 50 & $(58,8)$ & 35 & $(41,2)$ & \\
\hline Sim & 17 & $(16,7)$ & 2 & $(11,8)$ & 15 & $(88,2)$ & \\
\hline
\end{tabular}

${ }^{\mathrm{b}}$ Teste Exato de Fisher

Os dados dispostos na Tabela 5 demonstram que não foi verificada uma diferença significativa entre médias apresentados por idosos com e sem depressão no que se referem às saídas da instituição, as atividades físicas mensais, as atividades realizadas na instituição e os anos de internação e estudo.

Tabela 5 - Médias e desvio padrão entre idosos com e sem depressão residentes em instituições de longa permanência do Distrito Federal em 2010

\begin{tabular}{lccc}
\hline \multicolumn{1}{c}{ Variável } & $\begin{array}{c}\text { Sem Depressão } \\
\text { Média (DP) }\end{array}$ & $\begin{array}{c}\text { Com Depressão } \\
\text { Média (DP) }\end{array}$ & p \\
\hline Frequência anual de saída da instituição & $25,67(63,83)$ & $16,30(44,48)$ & $0,3934^{\mathrm{a}}$ \\
Frequência mensal da atividade física & $6,69(8,83)$ & $3,40(4,77)$ & $0,0935^{\mathrm{a}}$ \\
Frequência mensal de participação** & $3,54(4,86)$ & $2,88(3,84)$ & $0,5537^{\mathrm{a}}$ \\
Anos de instituição & $4,46(4,10)$ & $5,96(7,11)$ & $0,6561^{\mathrm{a}}$ \\
Anos de Estudo & $5,31(4,91)$ & $5,88(5,67)$ & $0,819^{\mathrm{a}}$ \\
\hline
\end{tabular}

DP: Desvio padrão. ${ }^{a}$ Mann-Whitney, ${ }^{* *} E m$ atividades da instituição

Os resultados da presente pesquisa foram apresentados às instituições participantes e aos profissionais responsáveis pela atenção à saúde e pelo cuidado dos pacientes, para melhor avaliação das condições clínicas, acompanhamento e melhoria da qualidade de vida de todos os idosos institucionalizados.

\section{DISCUSSÃO}

A depressão é a desordem psiquiátrica mais comum no idoso ${ }^{(2)}$, levando o indivíduo a perda da autonomia e ao agravamento de patologias preexistentes. Escalas de depressão para rastreamento de sintomas de depressão em idosos são 
relevantes, pois permitem a detecção prévia da depressão, possibilitando uma intervenção adequada, bem como a prevenção de fatores de risco ${ }^{(7)}$. No presente estudo foi realizado um rastreamento da presença de sinais indicativos de depressão e não de uma avaliação diagnóstica clínica.

Do total de idosos do presente estudo, 59,8\% não preencheram os critérios de inclusão e foram excluídos por não apresentaram condições clínicas para responder o questionário, devido à demência, rebaixamento do nível de consciência ou patologia psiquiátrica prévia. Entre diversos estudos no Brasil, a prevalência de doenças psiquiátricas entre idosos residentes em instituições de longa permanência variou entre $32,1 \%$ a $80,1 \%{ }^{(11)}$.

Resultados do projeto SABE (Saúde, Bem-Estar e Envelhecimento), da Organização Pan-Americana de Saúde, realizado com idosos residentes em áreas urbanas de metrópoles de sete países da América Latina e Caribe, verificaram presença de $18,1 \%$ de depressão, segundo a Escala de Depressão Geriátrica ${ }^{(12)}$. A prevalência de sintomas de depressão detectada no presente estudo $(49,0 \%)$ foi maior do que a relatada entre idosos de metrópoles da América Latina e Caribe ${ }^{(12)}$, e maior do que em um estudo na Inglaterra e País de Gales ${ }^{(13)}$ no qual foi verificada uma prevalência de depressão de $27,1 \%$ em pessoas que vivem em instituições em comparação com 9,3\% em pessoas que vivem em casa. Porém, foi semelhante a outro estudo nacional, utilizando-se o mesmo instrumento de avaliação, que verificou a prevalência de sintomas de depressão em $51 \%$ dos idosos institucionalizados ${ }^{(14)}$.

Com relação ao gênero, observou-se uma maior prevalência de sintomas de depressão entre mulheres $(p=0,018)$, fato que está de acordo com dados da literatura que sugerem serem as mulheres mais vulneráveis ao desenvolvimento de sintomas depressivos durante a veIhice ${ }^{(15)}$. Dentre as possíveis explicações está o fato de que as mulheres vivem, em média, mais do que os homens ${ }^{(16)} \mathrm{e}$ idades mais avançadas são acompanhadas por uma maior incidência de doenças crônicas, entre elas, a depressão(17).

O envelhecimento humano leva a uma diminuição progressiva da reserva funcional dos indivíduos que pode ser proporcional ao aumento da idade e a uma piora da qualidade de vida dos idosos, o que pode deixar o idoso mais suscetível a sintomas depressivos ${ }^{(18)}$. A associação

\section{REFERÊNCIA}

1. Avlund K, Lund R, Holstein BE, Due P. Social relations as determinant of onset of disability in aging. Arch Gerontol Geriatr. 2004;38(1):85-99.

2. Mann A. Depression in the elderly: findings from a community survey. Maturitas. 2001;38(1):53-8. entre a presença de depressão e a variável limitação/dependência foi significativa no presente estudo.

Observou-se associação positiva entre insatisfação do idoso e sintomas de depressão. A insatisfação pode ser explicada, em parte, pelo fato do idoso ser obrigado a conviver com desconhecidos, a seguir uma rotina de horários $^{(18)}$, perder parte de seu poder de escolha e o sentimento de ser apenas mais um dentro da coletividade ou da instituição ${ }^{(19)}$. Estes fatos podem levar ao aumento da incidência de depressão. Torna-se necessário então, durante o tratamento da depressão em idosos, compreender que as pessoas não vivenciam a depressão de maneira uniforme e que o cuidado deve ser dirigido para atender a sua singularidade de cada indivíduo(20).

A depressão costuma ser acompanhada por queixas físicas freqüentes ${ }^{(13)}$. Insônia, dor, parestesias, vertigem, suor excessivo e taquicardia tiveram associação importante e significativa com a depressão entre idosos.

Ao se identificar a depressão, uma equipe multiprofissional dever aturar para que um tratamento efetivo seja estabelecido. Nesse sentido, a enfermagem assume um papel fundamental, não devendo restringir-se aos cuidados relacionados ao tratamento medicamentoso, embora sejam importes. O estabelecimento de metas, a escuta, a interação com intuito de tornar o paciente consciente do seu papel no tratamento, na manutenção ou na supressão de sintomas, a produção de congruência perceptiva entre o enfermeiro e o paciente, onde o indivíduo compreende as intenções terapêuticas, além de perceber que o enfermeiro está ali para apoiá-lo, são atitudes esperadas dos enfermeiros que assistem pacientes com depressão(21-22).

\section{CONCLUSÃO}

O presente estudo verificou que os sintomas de depressão são freqüentes entre idosos institucionalizados, que estes ocorrem mais em mulheres, indivíduos com mais idade, com algum tipo de limitação/dependência e que estão insatisfeitos com a sua instituição. A observação de tais aspectos pode auxiliar o enfermeiro na proposição de ações preventivas, na identificação precoce da depressão, assim como no tratamento estabelecido em parceria com outros profissionais de saúde.
3. World Health Organization (WHO); World Organization of Family Doctors. Integrating mental health into primary care: a global perspective [Internet]. Geneva; 2008 [cited 2011 Dec 14]. Available from: http://whqlibdoc.who.int/publications/2008/9789241563680_eng.pdf

4. Gazalle FK, Hallal PC, Lima MS. Depressão na população idosa: os médicos estão investigando? Rev Bras Pisquiatr. 2004;26(3):145-9. 
5. Coutinho MPL, Gontiés B, Araújo LF, Sá RCN. Depressão um sofrimento sem fronteira: representações sociais entre crianças e idosos. Psicol USF. 2003;8(2):182-91.

6. Oliveira DAAP, Gomes L, Oliveira RF. Prevalência de depressão em idosos que freqüentam centros de convivência. Rev Saúde Pública. 2006;40(4):734-6.

7. Sousa RL, Medeiros JGM, Moura ACL, Souza CLM, Moreira IF. Validade e fidedignidade da Escala de Depressão Geriátrica na identificação de idosos deprimidos em um hospital geral. J Bras Psiquiatr. 2007;56(2):102-7.

8. Santana RF, Santos I. Como tornar-se idoso: um modelo de cuidar em enfermagem gerontológica. Texo Contexto Enferm. 2005;14(2):202-12.

9. Brum AKR, Tocantins FR, Silva TJES. O enfermeiro como instrumento de ação no cuidar do idoso. Rev Latino Am Enferm. 2005;13(6):1019-26.

10. Muñoz González LA, Romero YMP, López MR, Ramírez M, Stefanelli MC. The experience of family caregivers of older people with depression. Rev Esc Enferm USP [Internet]. 2010 [cited 2011 Dec 14];44(1):32-9. Available from: http:// www.scielo.br/pdf/reeusp/v44n1/en_a05v44n1.pdf

11. Gorzoni ML, Pires SL. Aspectos clínicos da demência senil em instituições asilares. Rev Psiquiatr Clín. 2006;33(1):18-23.

12. Lebrão ML, Laurenti R. Saúde, bem-estar e envelhecimento: o estudo SABE no Município de São Paulo. Rev Bras Epidemiol. 2005;8(2):127-41.

13. McDougall FA, Matthews FE, Kvaal K, Dewey ME, Brayne C. Prevalence and symptomatology of depression in older people living in institutions in England and Wales. Age Ageing. 2007;36(5):562-8.

14. Siqueira GR, Vasconcelos DT, Duarte GC, Arruda IC, Costa JAS, Cardoso RO. Análise da sintomatologia depressiva nos moradores do Abrigo Cristo Redentor através da aplicação da Escala de Depressão Geriátrica (EDG). Ciênc Saúde Coletiva. 2009;14(1):253-9.
15. Reynolds CF, Frank E, Dew MA, Houck PR, Miller M, Mazumdar S, et al. Treatment of 70-year olds with recurrent major depression. Am J Geriatr Psychiatry. 1999;7(1):64-9.

16. Karsch UM. Idosos dependentes: famílias e cuidadores. Cad Saúde Pública. 2003;19(3):820-9.

17. Leite VMM, Carvalho EMF, Barreto KML, Falcăo IV. Depressăo e envelhecimento: estudo nos participantes do Programa Universidade Aberta à Terceira Idade. Rev Bras Saúde Matern Infant. 2006;6(1):31-8.

18. Brasil. Ministério da Saúde; Secretaria de Atenção à Saúde. Envelhecimento e saúde da pessoa idosa [Internet]. Brasília; 2007 [citado 2011 dez. 14]. Disponível em: http://bvsms. saude.gov.br/bvs/publicacoes/abcad19.pdf

19. Cheloni CFP, Pinheiro FLS, Filho MC, Medeiros AL. Prevalência de depressão em idosos institucionalizados no município de Mossoró/RN segundo Escala de Depressão Geriátrica (Yesavage). Expressão. 2003;34(1-2):61-73.

20. Porcu M, Scantamburlo VM, Albrecht NR, Silva SP, Vallim FL, Araujo $\mathrm{CR}$, et al. Estudo comparativo sobre a prevalência de sintomas depressivos em idosos hospitalizados, institucionalizados e residentes na comunidade. Acta Sci. 2002; 24(3):713-7.

21. Gonçales CAV, Machado AL. Living with depression: women's life histories. Rev Esc Enferm USP [Internet]. 2008 [cited 2011 Dec 17];42(3):461-6. Available from: http://www.scielo.br/pdf/reeusp/v42n3/en_v42n3a06.pdf

22. Alligood MR. Family healthcare with King's Theory of goal attainment. Nurs Sci Q. 2010;23(2):99-104.

23. Furegato ARF, Nievas AF, Silva EC, Costa Jr ML. Pontos de vista e conhecimentos dos sinais indicativos de depressão entre acadêmicos de enfermagem. Rev Esc Enferm USP. 2005;39(4):401-8. 